\title{
A Promising New Approach for In Silico Prediction of Drug Concentration Profiles for Drug Candidates Lack of Experimental Pharmacokinetic Data
}

\author{
Jingchen Zhai ${ }^{1}$, Beihong $\mathrm{Ji}^{1}$, Shuhan $\mathrm{Liu}^{1}$, Yuzhao Zhang ${ }^{1}$, and Junmei Wang ${ }^{2}$ \\ ${ }^{1}$ University of Pittsburgh School of Pharmacy \\ ${ }^{2}$ University of Pittsburgh
}

June 2, 2020

\begin{abstract}
Aim: The purpose of this study is to develop a novel protocol to predict the concentration profiles of a target drug based on the PBPK model of a structurally similar template drug by combining two software for PBPK modeling, the SimCYP simulator and ADMET Predictor. Methods: The method was evaluated by utilizing 13 drug pairs which come from 18 drugs in the built-in database of the SimCYP software. All drug pairs have their Tanimoto scores no less than 0.5. Three versions (V1, V2 and V3) of models for the target drug were constructed by gradually replacing the corresponding parameters of the template drug with those predicted by ADME Predictor for the target drug. Normalized RMSE and Wilcoxon rank-sum test were introduced for the evaluation of the model performance. Results: Overall, V3 models demonstrated better performance than the V1 and V2 models did. The relationship between the model performance and structural similarity of drug pairs was also explored. Three protocols have come out as guidance on how to build PBPK models for target drugs: (1) V1 models are recommended when the structural similarity is very high; (2) V2 models are recommended when the similarity is below 0.65 or high than 0.85 ; (3) V3 models are recommended when the similarity is below 0.85. Conclusion: By leveraging the prediction accuracy and application practicality, this novel approach has a great promise in predicting the preliminary PK profiles for novel drugs, propelling the drug discovery process by suggesting drug candidates with promising PK profiles.
\end{abstract}

\section{Introduction}

Pharmacokinetics is the study of the time courses of a drug administered to the body, which includes the processes of absorption, distribution, metabolism and elimination (ADME). ${ }^{1}$ Usually it is essential to quantitatively measure the concentration of the drug in plasma at different time points in pharmacokinetic (PK) study, for the analysis of drug behavior and dose adjustment. In addition to clinical trials which always involved in time cost and ethical considerations, the "measurement" of concentration profiles under various administration conditions can also be achieved by the implementation of Physiologically based pharmacokinetic $(\mathrm{PBPK})^{2-4}$ modeling with known PK parameters related to drug properties or its ADME profiles. On the other hand, computational tools for both PBPK modeling and PK parameter prediction have been developed, further reducing experimental cost. Therefore, by virtue of such tools, the quick and convenient in silicoprediction of drug behavior in human body can be easily performed without investing much effort in experiments, informing further studies in drug toxicity, dosing strategy and potential drug-drug interactions. As such, this in silico method can be particularly useful in preclinical study and can serve as a tool to help select drug candidates which are more likely to have desirable PK profiles.

In this study, we developed a novel method to predict the concentration profile of a target compound based on PBPK models constructed using the model of a structurally similar drug which serves as the template. We utilized the SimCYP simulator (V19, Release 1; Shefeld, UK) ${ }^{5}$ software to construct PBPK models for a 
target drug by only substituting the predicted ADME parameters of the target drug for those applied by the PBPK model of the corresponding template drug. We applied ADMET Predictor (V9.5, Simulation Plus) , 6, 7 a software developed by SimulationPlus Inc. to predict the ADME properties of target drugs, which include physiochemical parameters like fraction unbound in plasma $\left(\mathrm{f}_{\mathrm{u}}\right)$ and blood-to-plasma partition ratio $(\mathrm{B} / \mathrm{P})$, and ADME input parameters such as volume of distribution $\left(\mathrm{V}_{\mathrm{d}}\right)$, Michaelis-Menten constant $\left(\mathrm{K}_{\mathrm{m}}\right)$ and maximal metabolism rate $\left(\mathrm{V}_{\max }\right)$ of common enzymes. Meanwhile, to better validate our constructed PBPK models as well as evaluate the performance of the two software tools, we selected 18 drugs (including substrates and inhibitors) collected by SimCYP compound library as the template drugs. In total, 13 drug pairs were formed based on their structural similarity. For each pair of drugs, one serves as the template and one as target drug. For the target drug in a drug pair, we pretended that no PBPK model was available for it and new PBPK models were constructed based on the PBPK model of the template drug. We tested three protocols by introducing ADME Predictor predicted ADME properties into the template PBPK model and evaluated the model performance using the observed PK profile of the target drug. The corresponding PBPK models constructed using the three protocols, in brief were called V1, V2 and V3 models, respectively.

\section{Methods}

\subsection{Drug preparation}

Drugs selected for the construction of in silico PBPK models come from the built-in drug database of the SimCYP software. Simplified Molecular-Input Line-Entry System (SMILES) ${ }^{8}$ strings of all drugs from SimCYP built-in library, including substrates and inhibitors, were collected from the DrugBank database (https://www.drugbank.ca/). The SMILES strings of drugs were used not only for their structural similarity calculation on a web platform, but also as inputs for the generation of their properties using ADMET Predictor.

\subsection{Structure similarity calculation}

Tanimoto scoring is a commonly used method to compute the fingerprint-based similarity between two compounds. ${ }^{9}$ In this study, we applied the maximum common substructure based (MCS) Tanimoto algorithm for the similarity calculation. The Tanimoto score (TS) is defined by the function below: ${ }^{10}$

$$
\operatorname{TS}(X, Y)=\frac{N_{Z}}{N_{X}+N_{Y}-N_{Z}}
$$

Where $\mathrm{N}_{\mathrm{X}}$ and $\mathrm{N}_{\mathrm{Y}}$ are the numbers of bits in fragment bit-strings of the two compounds, and $\mathrm{N}_{\mathrm{Z}}$ is the intersection set, i.e., the number of common substructures shared by these two compounds. TS $(\mathrm{X}, \mathrm{Y})$ ranges from 0 to 1 , measuring the structural similarity between two compounds from the lowest to the highest (when the two molecules are identical). TS scores were calculated using ChemMine (https://chemminetools.ucr.edu/similarity) for all combinations of drugs in the SimCYP compound database.

\subsection{Validation of PBPK models for drug templates}

We first validated the PBPK models of all selected 18 drugs by utilizing their observed data from literature. In detail, we utilized the original built-in models of those drugs in SimCYP to run the simulation. In terms of the trial design, the input dose, simulation time and population were the same as those reported in the clinical study of PK measurement. Meanwhile, the parameters of the built-in PBPK model, like the drug's ADME properties, remained the same for all the drugs except for Fluoxetine. As a racemate, we adjusted some of its ADME and PK parameters according to the literature to make the predicted curve much better fitting the experimental data. ${ }^{11-13}$ The key ADME parameters predicted by ADME Predictor for the 18 drugs were all listed in the Table $\mathbf{S 1}$, including the detail for the adjusted parameters of Fluoxetine. The observed drug concentration data of each template drug was extracted from published concentrationtime (C-T) curves using WebPlotDigitizer (https://automeris.io/WebPlotDigitizer/). The C-T curves from simulations were then overlaid to the observed drug concentrations. The predicted PK profiles of each 
template drug, including the maximal concentration $\left(\mathrm{C}_{\mathrm{Max}}\right)$, the time at which $\mathrm{C}_{\mathrm{Max}}$ is observed ( $\left.\mathrm{T}_{\mathrm{Max}}\right)$, and area under the curve (AUC), were compared to the observed ones.

\subsection{Model construction for target drugs}

In total, three versions of PBPK models for a target drug were built by modifying the models of the template drug: (1) in Version 1 (V1), only the molecular weight (MW) of template drug was changed to that of the target one; (2) in Version 2 (V2), in addition to the MW, the physiochemical properties, including $\mathrm{B} / \mathrm{P}$, $\mathrm{f}_{\mathrm{u}}$, octanol-buffer partition coefficient $\left(\log \mathrm{P}_{\mathrm{o}: \mathrm{w}}\right)$ value and acid dissociation constant $\left(\mathrm{pK}_{\mathrm{a}}\right)$ of the template drug were replaced by the ones predicted of the target drug; (3) in Version 3 (V3), in addition to MW and physiochemical properties, the input parameters for ADME process, including human jejunum effective permeability $\left(\mathrm{P}_{\text {eff }}\right)$ in absorption, $\mathrm{V}_{\mathrm{d}}$ in distribution, Cytochrome $\mathrm{P} 450(\mathrm{CYP})$ metabolism parameters $\left(\mathrm{K}_{\mathrm{m}}\right.$, $\mathrm{V}_{\max }$ or $\mathrm{CL}_{\text {int }}$ ) of templates were all replaced with the calculated ones for the target drug. All the ADME properties of the target drugs are predicted by ADMET Predictor, a software tool that can predict over 140 properties based on its built-in Quantitative structure-activity relationship (QSPR) models. ${ }^{14}$

\subsection{Evaluation of models for target drugs}

To evaluate performance of modified PBPK models for target drugs, the experimental data of target drugs were overlaid by the simulated C-T curves. To quantitively evaluate how well the experimental and simulated curves overlaid with each other, we calculated the root mean square error (RMSE) ${ }^{15}$ of the observed and predicted concentrations at different time points. The formula for the RMSE calculation is as follow:

$\mathrm{RMSE}=\left[\sum_{i=1}^{N}\left(C_{\mathrm{pi}}-C_{\mathrm{oi}}\right)^{2} / N\right]$

Where $\mathrm{C}_{\mathrm{oi}}$ and $\mathrm{C}_{\mathrm{pi}}$ represent the observed and predicted drug concentration at the time point i. $\mathrm{N}$ is the number of time points $(\mathrm{N}>1)$ from the extracted observed data. Specifically, in this study, to facilitate the comparison between models for different drugs with various concentration scales, we introduced normalized RMSE (NRMSE) to evaluate the performance of PBPK models, which is calculated using the following formula:

$\mathrm{NRMSE}=\frac{\mathrm{RMSE}}{C_{\max }-C_{\min }}$

Where $C_{\max }$ and $C_{\min }$ are the maximum and minimum values among the observed and predicted concentrations using all three versions of models.

We also conducted Wilcoxon rank-sum test ${ }^{16}$ to calculate the significance of difference between three versions of models based on the NRMSE results by using the SPSS software (Released 2019. IBM SPSS Statistics for Windows, Version 26.0. Armonk, NY: IBM Corp).

\section{Results}

\subsection{Drug pairs selection and validation of PBPK models for drug templates}

13 pairs out of 18 drugs, which have the calculated TS equal to or better than 0.5 , were selected for the in silico PBPK modeling. Drug pairs with TS below 0.5 were not considered to be structurally similar and were excluded in this study. The calculated TS for selected 13 pairs (Groups A-M) were listed in Table 1 . Since both drugs in a pair will in turn serve as the template and target drug for cross validation, we used $\mathrm{X}-1$ and $\mathrm{X}-2$ to label two drugs in the pair, respectively, where $\mathrm{X}$ can be A to M.

The predicted mean plasma concentration-time profiles overlaid with observed data of all 18 template drugs are shown in Figure 1. Accordingly, Table 2 exhibits the predicted PK parameters $\left(\mathrm{C}_{\mathrm{Max}}, \mathrm{T}_{\mathrm{Max}}, \mathrm{AUC}\right)$ versus observed values. From Table 2 , excluding the drugs with observed PK parameters all unavailable (Mephenytoin and Fluoxetine), the predicted PK parameters of most drugs are within the standard deviation ranges of their observed values. The predicted values of $\mathrm{C}_{\mathrm{Max}}, \mathrm{T}_{\mathrm{Max}}$ and $\mathrm{AUC}$ for Theophyline are all slightly beyond the margin of error but still within the range of two-fold standard deviation. Overall, as shown in Figure 1, the observed C-T profiles are within the 95\% Confidence Interval (CI) ranges (the upper and 
lower grey dashed curves) of the simulated C-T curves. Therefore, the PBPK models for the template drugs have been well validated.

\subsection{Predicted concentration profiles for the in silicoPBPK models}

The C-T profiles predicted by all three versions (Versions 1, 2, and 3) of PBPK models are shown in Figure 2 . To quantitatively measure the deviation of predicted concentration profiles from the experimental data, the difference between observed and predicted values of three versions were respectively evaluated by NRMSE (Table 3 ). The lower the NRMSE value is, the smaller the difference between the predicted and experimental concentration profile is, i.e., the better performance the created model for target drug is. Overall, among the 26 PBPK models constructed using the V3 computational protocol, more than a half of them showed satisfactory performance. Specifically, in Groups C and G-J, the predicted performance of V3 models for all drug pairs outperformed the corresponding V1 and V2 models. For Groups B, D-F and K-L, although the performance of V3 models is not the best, the predicted NRMSE values are close to those of V1 or V2 models. For D-2 drug pair, though the NRMSE of the V2 model is the lowest, the predicted C-T curve by the V3 model has a better shape fitting the observed data as shown in Figure 2 . However, for some drug pairs, like Groups A and M, the performance of the V3 models is inferior to that of the V1 and V2 models. Interestingly, Group A has the smallest and Group M has the largest structural similarity among all the 13 pairs. The predicted PK parameters, $\mathrm{C}_{\mathrm{Max}}, \mathrm{T}_{\mathrm{Max}}$ and AUC, are listed in Table S2 .

Based on NRMSE, a pure in silico PBPK model in this study can be classified into different categories: excellent (NRMSE [?] 0.1), good (0.1 < NRMSE [?] 0.2), average $(0.2<$ NRMSE [?] 0.6) and poor (NRMSE > 0.6). As indicated in Figures 3-4 andTable 3, one V1, three V2 and five V3 out of 26 models belong to the "excellent" category. Nearly half of V2 and V3 models belong to the first two categories, while only 5 V1 models belong to the first two category. For the last category, there are 7, 3 and 1 models for V1, V2 and V3, respectively. In summary, the performance of V3 models is slightly better than that of V2, but much better than that of V1.

\subsection{Statistical significance of the performance difference between three versions of models}

The Wilcoxon rank-sum test was conducted to further demonstrate the NRMSE variations between predicted performance of modified models in three versions. The significance of difference between different model versions was evaluated by p-values. According to the significance test, there is a significant improvement on performance of V3 models ( $\mathrm{p}$-value $=0.043$ ) compared to V1 models, as there are 17 out of 26 pure in silico PBPK models show the lower NRMSE in V3, whereas, only 9 V1 models have the lower NRMSE values. There is no significant difference between the NRMSE of V2 and V1 models (p-value $=0.069$ ), as well as V3 and V2 models $(p-v a l u e ~=0.328)$. Although in comparison with V1, there are 16 V2 models with lower NRMSE, while only 10 V1 models have lower NRMSE values. Similarly, 16 V3 models have lower NRMSE than V2 models, whereas $10 \mathrm{~V} 2$ models display lower NRMSE values.

\section{Discussion}

In this study, we developed a novel approach to construct in silico PBPK models for target drugs lack of experimental ADME and other PK parameters using an established PBPK model of a structurally similar drug as the model template. We used 18 drugs which formed 13 drug pairs (Groups A-M) for which the structural similarity is equal to or larger than 0.5 to evaluate three ADME parameter substitution protocols, which are corresponding to three versions of PBPK models. The performance of the in silico PBPK models were critically evaluated using experimental PK profiles and parameters.

\subsection{The relationship between similarity and model performance}

We attempted to obtain guidance on selecting suitable template drug for a given target drug. We focused on using structural similarity to select the template drugs. For this purpose, we divided the 18 pairs of drugs into three categories based on their structural similarity. Groups A-E belong to the first category and their TS are not higher than 0.65; Groups F-J belong to the second category and their TS range from 0.65 to 0.85 ; and Groups K, L and M belong to the third category with TS larger than 0.85. For the 
first category, the mean calculated NRMSEs are $0.42,0.35$ and 0.34 for V1, V2 and V3, respectively. This result suggests that the performance of V3 and V2 are comparable and marginally better than that of V1 for low structural similarity drug pairs. For the second category, the V3 models, who's mean NRMSE is 0.18 , apparently outperformed the other two versions of models (NRMSE are 0.54 and 0.41 for V1 and V2 models, respectively). As to the high structural similarity pairs in the last category, the overall performance of all the three models are comparable and NRMSE are 0.28, 0.22 and 0.30 for the three versions of models correspondingly. This observation can be explained as follows: when drug pairs have very high structural similarity ( $>0.85$ ), the ADME properties are very similar between the target and template drugs, the V3 protocol actually introduces more errors by substitution of the predicted ADME properties with ADME Predictor for the corresponding parameters in the template model.

Based on the above analysis, the first modeling protocol for constructing V1 models is recommended when the structural similarity is very high (Group M); the second modeling protocol for constructing V2 models is recommended when the structural similarity is below 0.65 or high than 0.85 ; while the third protocol for constructing V3 models are recommended when structural similarity is below 0.85 . It is of note that this is just a general guidance and there are many special cases not obeying those rules. In real practice, it is recommended to construct the V3 model when the structural similarity is smaller than 0.85 , and V2 model otherwise.

\subsection{Perspective of applying in silico PBPK modeling for compounds lack of experimental ADME and PK properties}

In this study, we put forward a novel approach to build PBPK models for a target drug which is lack of measured ADME and PK parameters using the PBPK model of a template drug which is structurally similar to the target drug. Also, we proposed an overall guidance on selecting suitable template drug and using its PBPK model as the model template. The success of this computational approach depends on two important factors, the availability of high quality PBPK model for the template compound and the accuracy and consistency of the ADME and PK parameters predicted by ADME Predictor software for the target drug. Additionally, not all the ADME/PK properties can be calculated with the current version of ADME Predictor. For example, the prediction of metabolism in ADMET Predictor is only limited to 5 commonly used enzymes (CYP1A2, CYP2D6, CYP2C9, CYP2C19 and CYP3A4). Nevertheless, we have proposed a practical approach to generate PBPK models for a compound lack of experimental ADME/PK properties. This model can serve as the initial version of the PBPK models for the target compound, and its performance can be improved using the measured PK profiles and properties in the future. The computational protocol introduced in this work may have great applications in selecting drug leads to enter the drug optimization phase or drug candidates to enter preclinical studies.

\subsection{Significance and future work}

In this work, we have introduced and tested a novel computational protocol to develop in silico PBPK model for a compound lack of measured ADME/PK properties and PK profiles. The general idea is to choose a proper PBPK model as the template, when the corresponding compound, the template drug, is structurally similar to the target drug. For the target drug, we calculated the ADME properties using ADME Predictor of SimulationPlus Inc. We have evaluated three versions of substitution to introduce calculated ADME properties for the target molecule to the template PBPK model using 13 drug pairs, and for the involved drugs, the measured PK properties and the C-T profiles are available. We have come out an overall guidance on how to build PBPK models for target drugs based on structural similarity, i.e. if the Tanimoto score is smaller than 0.85 , the V3 version is recommended while V2 protocol is recommend for structurally highly similar compounds (TS $>0.85$ ). Following this guidance, the mean NRMSE of 26 PBPK models is 0.25 , better than any versions of the models, which are $0.43,0.34$ and 0.27 for V1, V2 and V3, respectively. While future experimental work is definitely needed to further improve the model performance, our novel approach can help identify drug candidates with favorable PK profiles, reducing experimental cost and providing insight in drug discovery and development. 


\section{Supporting Information}

The input parameters of 18 drugs and predicted PK profiles of models in V1-V3 are listed in Tables S1-S2

\section{Competent interest}

All authors declare no conflict of interest.

\section{Acknowledgement}

The author gratefully acknowledges the funding support from the National Institutes of Health (R01GM079383, R21GM097617 and P30DA035778).

\section{Reference}

1. Eddershaw PJ, Beresford AP, Bayliss MK. ADME/PK as part of a rational approach to drug discovery. Drug Discovery Today . 2000/09/01/ 2000;5(9):409-414. doi:https://doi.org/10.1016/S1359-6446(00)015403

2. Lu J, Goldsmith M-R, Grulke CM, et al. Developing a physiologically-based pharmacokinetic model knowledgebase in support of provisional model construction. PLoS computational biology . 2016;12(2)

3. Zhuang X, Lu C. PBPK modeling and simulation in drug research and development. Acta Pharmaceutica Sinica B . 2016;6(5):430-440.

4. Lin L, Wong H. Predicting oral drug absorption: mini review on physiologically-based pharmacokinetic models. Pharmaceutics . 2017;9(4):41.

5. Jamei M, Marciniak S, Feng K, Barnett A, Tucker G, Rostami-Hodjegan A. The Simcyp@ Populationbased ADME Simulator. Expert Opinion on Drug Metabolism 83 Toxicology . 2009/02/01 2009;5(2):211-223. doi:10.1517/17425250802691074

6. Hassan SF, Rashid U, Ansari FL, Ul-Haq Z. Bioisosteric approach in designing new monastrol derivatives: an investigation on their ADMET prediction using in silico derived parameters. Journal of Molecular Graphics and Modelling . 2013;45:202-210.

7. Hecht D, Fogel GB. Computational intelligence methods for ADMET prediction. Front Drug Des Discov . 2009;4:351-377.

8. Toropov AA, Toropova AP, Mukhamedzhanoval DV, Gutman I. Simplified molecular input line entry system (SMILES) as an alternative for constructing quantitative structure-property relationships (QSPR). 2005 ;

9. Bajusz D, Rácz A, Héberger K. Why is Tanimoto index an appropriate choice for fingerprint-based similarity calculations? Journal of cheminformatics . 2015;7(1):20.

10. Sharma A, Lal SP. Tanimoto based similarity measure for intrusion detection system. Journal of Information Security . 2011;2(04):195.

11. Agoram B, Woltosz WS, Bolger MB. Predicting the impact of physiological and biochemical processes on oral drug bioavailability. Advanced drug delivery reviews . 2001;50:S41-S67.

12. Franceschi L, Faggiani A, Furlanut M. A simple method to monitor serum concentrations of fluoxetine and its major metabolite for pharmacokinetic studies. Journal of pharmaceutical and biomedical analysis . 2009;49(2):554-557.

13. D'Souza DL, Dimmitt DC, Robbins DK, Nezamis J, Simms L, Koch KM. Effect of alosetron on the pharmacokinetics of fluoxetine. The Journal of Clinical Pharmacology . 2001;41(4):455-458. 
14. Tropsha A, Gramatica P, Gombar VK. The importance of being earnest: validation is the absolute essential for successful application and interpretation of QSPR models. QSAR \& Combinatorial Science . 2003;22(1):69-77.

15. Chai T, Draxler RR. Root mean square error (RMSE) or mean absolute error (MAE)? GMDD . 2014;7(1):1525-1534.

16. Rosner B, Glynn RJ, Ting Lee ML. Incorporation of clustering effects for the Wilcoxon rank sum test: a large-sample approach. Biometrics . 2003;59(4):1089-1098.

17. Connarn JN, Flowers S, Kelly M, et al. Pharmacokinetics and pharmacogenomics of bupropion in three different formulations with different release kinetics in healthy human volunteers. The AAPS journal . 2017;19(5):1513-1522.

18. East T, Dye D. Determination of dextromethorphan and metabolites in human plasma and urine by highperformance liquid chromatography with fluorescence detection. Journal of Chromatography B: Biomedical Sciences and Applications . 1985;338:99-112.

19. Pringle T, Francis R, East P, Shanks R. Pharmacodynamic and pharmacokinetic studies on bufuralol in man. British journal of clinical pharmacology . 1986;22(5):527-534.

20. Kolb KW, Garnett WR, Small RE, Vetrovec GW, Kline BJ, Fox T. Effect of cimetidine on quinidine clearance. Therapeutic drug monitoring . 1984;6(3):306-312.

21. Chen X, Jacobs G, de Kam M, et al. The central nervous system effects of the partial GABA-A $\alpha 2$, 3 -selective receptor modulator AZD7325 in comparison with lorazepam in healthy males. British journal of clinical pharmacology . 2014;78(6):1298-1314.

22. Link B, Haschke M, Grignaschi N, et al. Pharmacokinetics of intravenous and oral midazolam in plasma and saliva in humans: usefulness of saliva as matrix for CYP3A phenotyping. British journal of clinical pharmacology . 2008;66(4):473-484.

23. Otani K, Yasui N, Furukori H, et al. Relationship between single oral dose pharmacokinetics of alprazolam and triazolam.International clinical psychopharmacology . 1997;12(3):153-157.

24. Greenblatt DJ, Harmatz JS, von Moltke LL, et al. Comparative kinetics and response to the benzodiazepine agonists triazolam and zolpidem: evaluation of sex-dependent differences. Journal of Pharmacology and Experimental Therapeutics . 2000;293(2):435-443.

25. Wedlund P, Aslanian W, Jacqz E, McAllister C, Branch R, Wilkinson G. Phenotypic differences in mephenytoin pharmacokinetics in normal subjects. Journal of Pharmacology and Experimental Therapeutics . 1985;234(3):662-669.

26. NELSON E, POWELL JR, CONRAD K, et al. Phenobarbital pharmacokinetics and bioavailability in adults. The Journal of Clinical Pharmacology . 1982;22(2-3):141-148.

27. Chalon SA, Desager JP, DeSante KA, et al. Effect of hepatic impairment on the pharmacokinetics of atomoxetine and its metabolites. Clinical Pharmacology \& Therapeutics . 2003;73(3):178-191.

28. Sugimoto Ki, Ohmori M, Tsuruoka S, et al. Different effects of St John's wort on the pharmacokinetics of simvastatin and pravastatin. Clinical Pharmacology 83 Therapeutics . 2001;70(6):518-524.

29. Qiu F, Wang G, Zhao Y, Sun H, Mao G, Sun J. Effect of danshen extract on pharmacokinetics of theophylline in healthy volunteers. British journal of clinical pharmacology . 2008;65(2):270-274.

30. Laizure SC, Meibohm B, Nelson K, Chen F, Hu ZY, Parker RB. Comparison of caffeine disposition following administration by oral solution (energy drink) and inspired powder (AeroShot) in human subjects. British journal of clinical pharmacology . 2017;83(12):2687-2694. 
31. Ciraulo DA, Barnhill JG, Jaffe JH. Clinical pharmacokinetics of imipramine and desipramine in alcoholics and normal volunteers. Clinical Pharmacology $\&$ Therapeutics . 1988;43(5):509-518.

32. Madani S, Barilla D, Cramer J, Wang Y, Paul C. Effect of terbinafine on the pharmacokinetics and pharmacodynamics of desipramine in healthy volunteers identified as cytochrome P450 2D6 (CYP2D6) extensive metabolizers. The Journal of Clinical Pharmacology . 2002;42(11):1211-1218.

\section{Hosted file}

Title_page.docx available at https://authorea.com/users/328868/articles/456012-apromising-new-approach-for-in-silico-prediction-of-drug-concentration-profiles-fordrug-candidates-lack-of-experimental-pharmacokinetic-data

\section{Hosted file}

Tables.docx available at https://authorea.com/users/328868/articles/456012-a-promising-newapproach-for-in-silico-prediction-of-drug-concentration-profiles-for-drug-candidateslack-of-experimental-pharmacokinetic-data
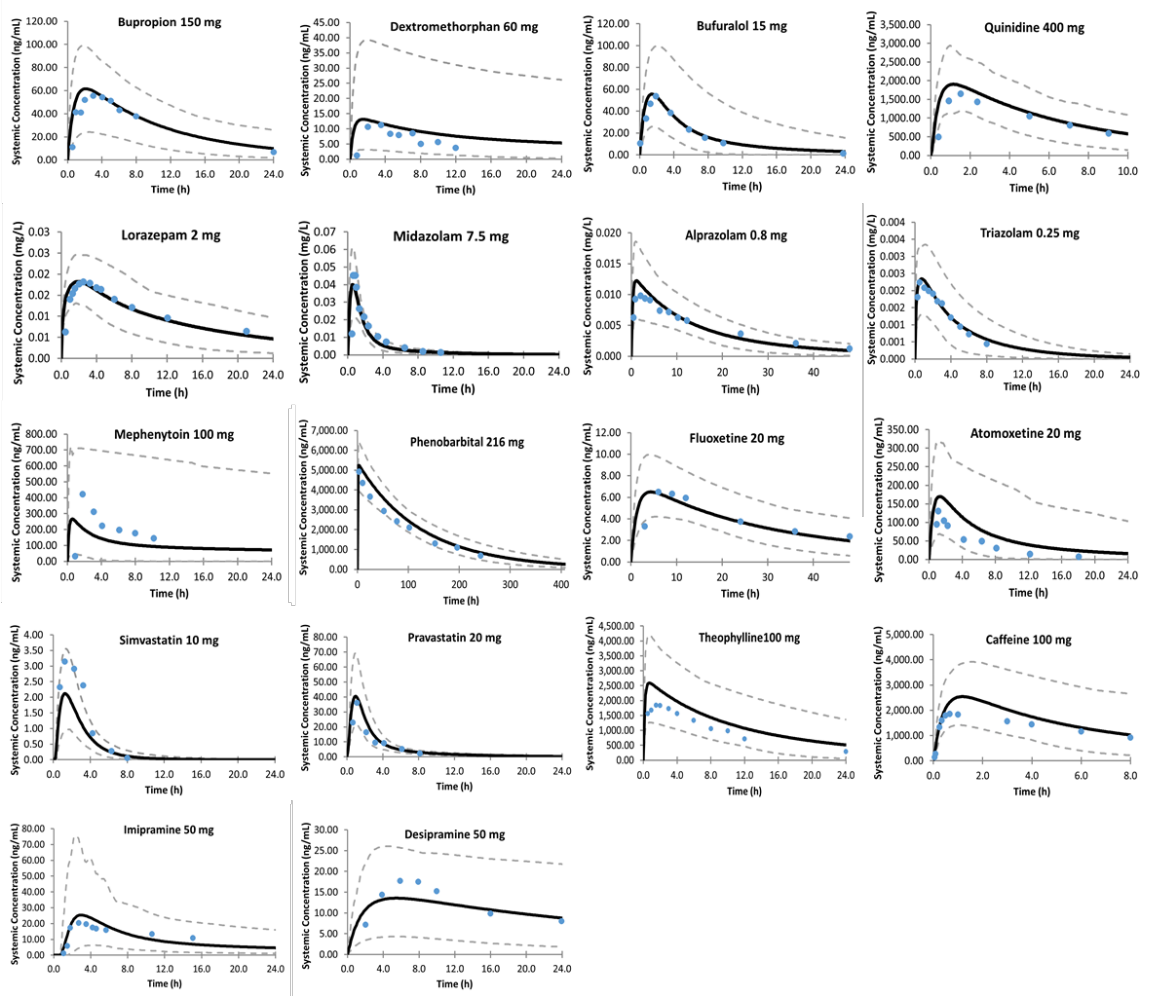

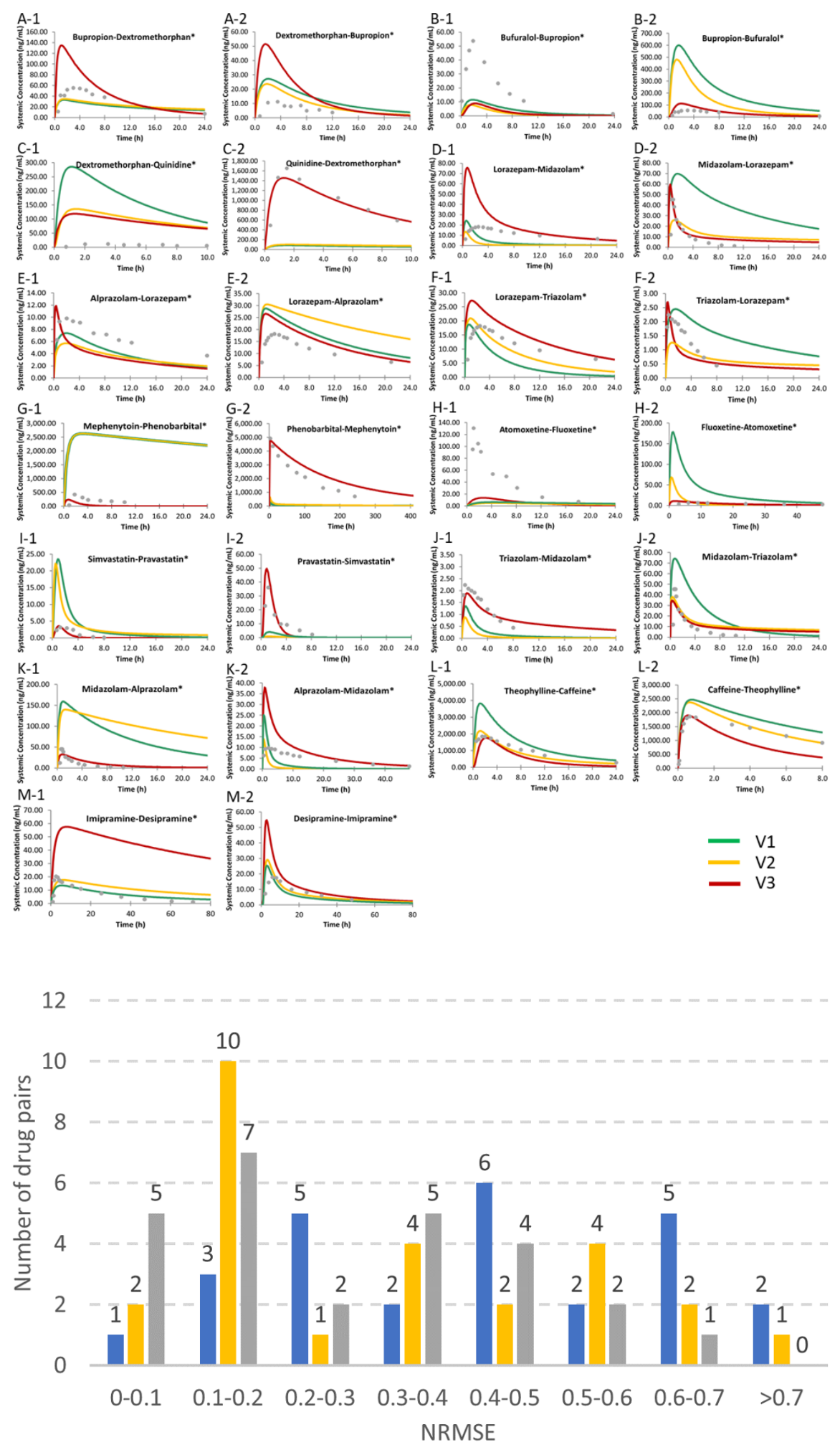

$\square \mathrm{V} 1 \backsim \mathrm{V} 2 \backsim \mathrm{V} 3$ 


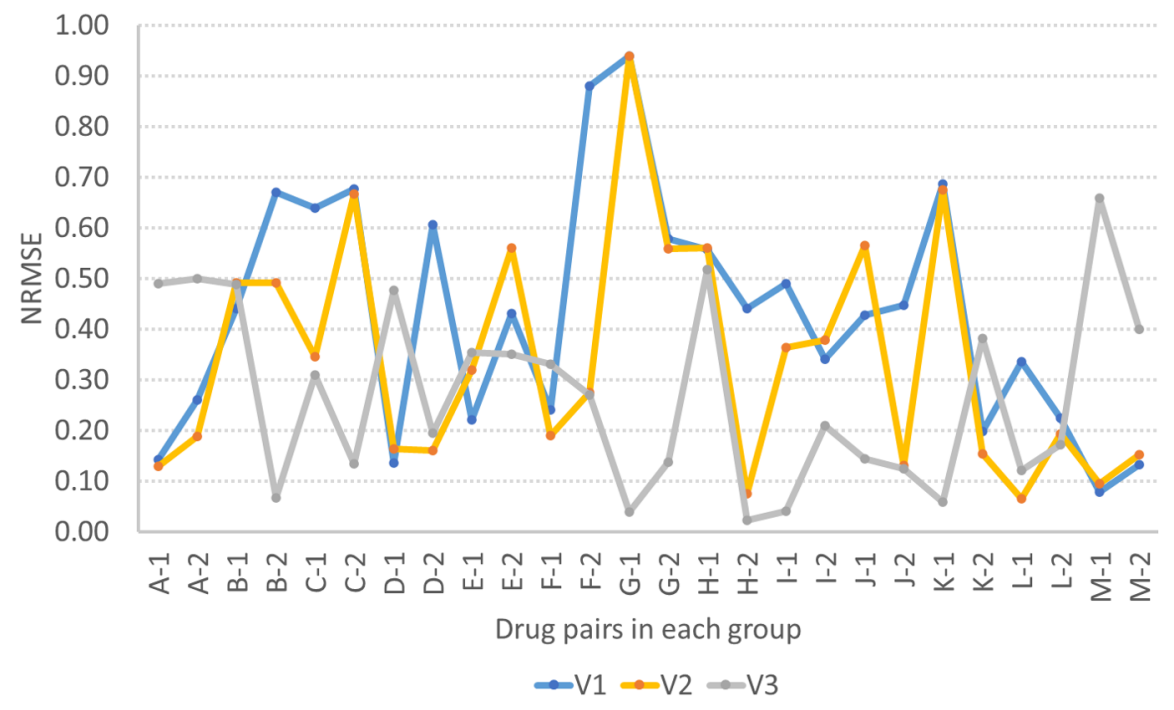

